Review

\title{
Polatuzumab Vedotin: Current Role and Future Applications in the Treatment of Patients with Diffuse Large B-Cell Lymphoma
}

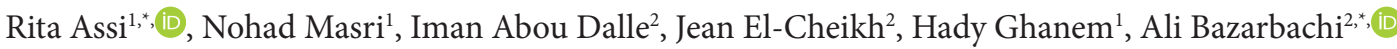 \\ ${ }^{1}$ Department of Internal Medicine, Division of Hematology-Oncology, Lebanese American University and Lebanese American University \\ Medical Center-Rizk Hospital, Beirut, Lebanon \\ ${ }^{2}$ Department of Internal Medicine, Division of Hematology-Oncology, American University of Beirut Medical Center, Beirut, Lebanon
}

\section{ARTICLE INFO}

Article History

Received 09 February 2021

Accepted 26 February 2021

\section{Keywords}

DLBCL

relapsed/refractory

transplantation

ADC

polatuzumab vedotin

\begin{abstract}
Diffuse large B-cell lymphoma (DLBCL) is a biologically and clinically heterogeneous disease. Despite good responses to standard of care frontline chemoimmunotherapy, the prognosis of relapsed/refractory $(\mathrm{R} / \mathrm{R})$ patients remains obscured by the possible inadequate responses to salvage therapy, eligibility for autologous transplantation, age and comorbidities. Polatuzumab vedotin is an antibody-drug conjugate formed by a CD79b antibody conjugated to the highly cytotoxic agent monomethyl auristatin $\mathrm{E}$ by means of a cleavable linker. Following significant clinical efficacy in R/R DLBCL, polatuzumab vedotin was granted accelerated Food and Drug Administration (FDA) approval in combination with bendamustine plus rituximab for patients who have failed at least two prior therapies. Other clinical studies involving polatuzumab vedotin in combination with other therapy regimens are also under evaluation for previously untreated DLBCL patients. In this article, we review the different phases from the preclinical development of polatuzumab vedotin to studies leading to its first approval, and highlight the potential future roles of this molecule in the treatment landscape of DLBCL.
\end{abstract}

(c) 2021 International Academy for Clinical Hematology. Publishing services by Atlantis Press International B.V. This is an open access article distributed under the CC BY-NC 4.0 license (http://creativecommons.org/licenses/by-nc/4.0/).

\section{INTRODUCTION}

Diffuse large B-cell lymphomas (DLBCL) constitute around 30\% of all non-Hodgkin lymphomas (NHL) and account for the largest group of aggressive lymphomas [1]. DLBCL are further subdivided into three molecular subtypes by cell-of-origin (COO) portending prognostic and therapeutic implications: the germinal center B-cell phenotype (GCB), the activated peripheral B-cell subtype (ABC) and unclassifiable category [2]. The current standard of care frontline chemotherapy regimen includes the use of cyclophosphamide, doxorubicin, vincristine, and prednisone (CHOP), along with rituximab, a monoclonal antibody (MoAb) against the surface antigen CD20. Indeed, the advent of rituximab in the late 1990s, and its addition to $\mathrm{CHOP}$ (R-CHOP) [3] resulted in improved outcomes in all disease stages and settings with current 4 -year overall survival (OS) ranging from $55 \%$ to $95 \%$ and a cure rate of around $60 \%$ [4]. Nevertheless, the clinical course can be life-threatening, with up to $40 \%$ of patients who can still relapse or have refractory disease, a scenario resulting in 5-year survival rates as low as approximately $25 \%$ for high-risk patients [5]. Subsequent outcomes differ greatly between patients depending on their transplant eligibility and response to second-line treatment. Multiple salvage regimens have been used, including dexamethasone, cytarabine and platinum,

"Corresponding authors. Email: rita.assi@laumcrh.com; bazarbac@aub.edu.lb Peer review under responsibility of the International Academy for Clinical Hematology ifosfamide, carboplatin and etoposide or gemcitabine, dexamethasone and platinum (GDP), followed by autologous peripheral stem cell transplantation (APSCT), resulting in cure rates approaching $30-40 \%[6]$.

These aggressive approaches are unfortunately limited to relatively younger candidates, leaving older patients and those who relapse post-APSCT with fewer treatment options and succumbing to their disease. In the absence of a current role for maintenance therapy in DLBCL, second-line therapies and beyond for non-APSCT eligible patients include the use of gemcitabine-oxaliplatin combination, bendamustine with or without rituximab, as well as lenalidomide and ibrutinib for non-GCB molecular subtypes [7].

\section{THE EMERGENCE OF NOVEL AGENTS}

To cater for this unmet clinical need in the relapsed/refractory (R/R) DLBCL setting, novel agents are being studied. More recently, CD19-directed chimeric antigen receptor T-cell (CAR-T) therapy has been approved for the R/R cases and those who relapse or achieve partial remission after second line treatment $[8,9]$. However, its use remains restricted due to toxicity, availability, and prohibitive cost.

One promising strategy involves the use of antibody-drug conjugates (ADCs). These smart compounds combine the highly specific 
targeting potential of a MoAb toward a tumor-associated antigen with the cytotoxic function of a small active potent toxin, all joined by a bio-cleavable chemical linker, and exhibit a favorable pharmacokinetic profile [10]. The complex is internalized and the linker is degraded once the MoAb binds the antigen, therefore releasing the cytotoxic payload within the tumoral cell and resulting in its death [11]. Polatuzumab vedotin (polatuzumab vedotin-piiq; Polivy ${ }^{\mathrm{Tm}}$, Roche, Basel, Switzerland) represents a prototypic and novel ADC consisting of a MoAb against CD79b [a specific B-cell receptor (BCR)] covalently linked to the anti-mitotic cytotoxic agent monomethyl auristatin (MMAE) through a citrulline-valine cleavable peptide linker that is stable in plasma. Once released, MMAE causes microtubule disruption, thereby inhibiting cellular division and inducing apoptosis of proliferating B-cell. Interestingly, polatuzumab vedotin carries an average of 3.5 molecules of MMAE for each CD79b MoAb [12].

The number of available therapy options for patients with $R / R$ DLBCL may be enthusiastically expanding with the recent FDA approval of polatuzumab vedotin for use in combination with bendamustine and rituximab for the treatment of patients with $R / R$ DLBCL who have received at least two prior therapies. In this review, we outline the different milestones from the rationale and development of polatuzumab vedotin, its approval as well as ongoing studies and future perspective.

\section{RATIONALE AND DEVELOPMENT OF POLATUZUMAB VEDOTIN}

\subsection{CD79b: The Promising Target}

CD79 is a heterodimer molecule involved in signal transduction and composed of $\mathrm{CD} 79 \mathrm{a}$ and $\mathrm{CD} 79 \mathrm{~b}$ ( $\operatorname{Ig} \alpha$ and $\operatorname{Ig} \beta$ respectively) expressed almost exclusively on B-cells and B-cell neoplasms. CD79 is non-covalently linked to the membrane immunoglobulin (IgM) and the group constitutes the BCR [13]. During B-cell ontogeny and differentiation, CD79a and CD79b are expressed in early stages, preceding the immunoglobulin heavy-chain gene rearrangement and CD20 expression but disappear later than CD20 in the late (plasma cell) stage [14]. Therefore, antibodies to CD79a and CD79b can be useful in the differential diagnosis of B-cell from T-cell neoplasms or myeloid neoplasms, or L- and H-lymphocyte predominant Hodgkin's lymphoma from classical Hodgkin's lymphoma. More specifically, CD79b was found to play an essential role in the BCR expression, transport and functionality by providing signaling capacity to regulate processes such as allelic exclusion, proliferation, differentiation, anergy, and apoptosis $[15,16]$. These observations further established CD79b as an optimal target for selective immunotherapy in the treatment of B-cell malignancies.

\subsection{Preclinical Data}

A study by Dornan et al. [17] initially established that a minimal expression of CD79b by flow cytometry was sufficient for the in vitro activity of anti-CD79b ADC in NHL cell lines, and that this expression persisted in the majority of relapsed DLBCL cases, irrespective of the COO subtype. Preclinical data later suggested that polatuzumab vedotin is active against various DLBCL cell lines, including $\mathrm{ABC}, \mathrm{GCL}$, and even those harboring mutations in $C D 79 b$ shown to confer poor survival in DLBCL [18]. Furthermore, these DLBCL cell lines displayed global sensitivity to polatuzumab vedotin despite the expression of several proteins which have adverse impact on survival and/or responses to chemotherapy, including BCL-XL and MYC, as well as BCL2 and MCL1 to a lesser extent [18]. Of note, somatic mutations of $C D 79 b$ enhance the surface BCR expression and attenuate Lyn kinase (165120), a known feedback inhibitor of BCR signaling [15]. These landmark findings establish chronic active BCR signaling as a new pathogenic mechanism in ABC subtype DLBCL, thereby reinforcing the potentially promising benefits of polatuzumab vedotin in this specific setting.

Polatuzumab vedotin also decreased cell proliferation and enhanced cell death of CD79b+ aggressive B-cell NHL cell lines [19], translating into improved OS in their murine xenograft models [20]. The anti-neoplastic effects of polatuzumab vedotin could be further improved when combined with other active agents such as venetoclax [21] and obinutuzumab [22], further broadening its future potential therapeutic indications.

\section{EARLY CLINICAL TRIALS}

\subsection{Monotherapy}

A phase I open-label trial (NCT01290549) evaluated the use of polatuzumab vedotin as single-agent in patients with $\mathrm{R} / \mathrm{R}$ B-cell NHL administered intravenously in 21-day cycles [23]. In the dose-escalation cohort of 34 NHL patients, PV was used at doses of $0.1-2.4 \mathrm{mg} / \mathrm{kg}$, where $2.4 \mathrm{mg} / \mathrm{kg}$ was established as the recommended phase II dose (RP2D) based on meticulous pharmacokinetics, safety tolerability, and recorded dose-limiting toxicities. Of the 42 evaluable patients with NHL who received polatuzumab vedotin at $2.4 \mathrm{mg} / \mathrm{kg}$ in the escalation or expansion phases, 23 had an objective response (OR) with seven complete responses (CR) and 16 partial responses (PR). Importantly, these responders included 14 of 25 patients with DLBCL (4 CRs; 10 PRs). Overall, patients receiving polatuzumab vedotin at $2.4 \mathrm{mg} / \mathrm{kg}$ recorded a median duration of response (DoR) of 6.2 months and a median progression-free survival (PFS) of 5.7 months. A similarly designed Japanese phase I trial (JO29138) of patients with R/R B-cell NHL using polatuzumab vedotin at 1.0 or $1.8 \mathrm{mg} / \mathrm{kg}$ every 21 days confirmed the same findings [24].

\subsection{Combination with Immunotherapy}

In the phase II part of the randomized, open-label ROMULUS phase Ib/II trial (NCT01691898), polatuzumab vedotin $2.4 \mathrm{mg} / \mathrm{kg}$ was subsequently evaluated in combination with rituximab in patients with $\mathrm{R} / \mathrm{R}$ DLBCL $(n=39)$ or follicular lymphoma (FL; $n=20)$ [25]. In that study, polatuzumab vedotin or pinatuzumab vedotin were administered with rituximab every 21 days until progressive disease or unacceptable toxicity, for up to 1 year. Interestingly, enrolled patients had failed up to four prior systemic therapies, with $78 \%$ of DLBCL patients being refractory to their last treatment [25]. DLBCL patients exhibited a CR rate of $21 \%$, PR of $33 \%$, leading to an OR of $54 \%$ and a median DoR of 13.4 months. Additionally, these patients had a median PFS of 5.6 months while the median OS reached 20.1 months. In the 31 patients who were 
refractory to their last treatment, the OR was $45 \%$, the median DoR was 13.4 months, while the median PFS and OS were 4.7 and 11.7 months, respectively.

It is important to note that the efficacy results of polatuzumab vedotin plus rituximab were overall similar to those of the anti-CD22 ADC pinatuzumab vedotin used with rituximab, except for a numerical difference between the regimens in terms of the median DoR in DLBCL patients (13.4 versus 6.2 months, respectively) favoring polatuzumab. The combination of polatuzumab vedotin plus rituximab demonstrated clinical activity regardless of DLBCL COO subtypes, as well as in those expressing high levels of BCL-2. Nevertheless, the levels of BCL-2 or CD79b expression did not predict the degree of tumor shrinkage in these patients [25].

The ROMULUS trial also evaluated the addition of polatuzumab vedotin $1.8 \mathrm{mg} / \mathrm{kg}$ to obinutuzumab in 21 patients with $\mathrm{R} / \mathrm{R}$ DLBCL for up to eight 21-day cycles [26]. In this cohort, patients had failed 1-8 prior therapies, with the majority (61\%) being refractory to the last. At the most recent follow-up, the combination showed a preliminary OR of $53 \%$ (29\% CR, 24\% PR) as assessed by modified Lugano 2014 criteria.

\subsection{Combination with Chemotherapy}

The aforementioned trials demonstrated encouraging results of polatuzumab vedotin as monotherapy [23] and in combination with an anti-CD20 MoAb [25], in terms of efficacy, safety profile and survival outcomes in transplant ineligible patients with heavily pretreated R/R DLBCL.

In an effort to improve the modest depth as well as duration of responses, polatuzumab vedotin was subsequently evaluated with bendamustine and either rituximab (P-BR) or obinutuzumab for the treatment of R/R DLBCL or FL in an open-label multicenter ongoing phase Ib/II trial (NCT02257567; GO29365) [27] that led to its accelerated FDA approval on June 10, 2019 for use in this setting. While patients with double- and triple-hit lymphomas were allowed, none were actually enrolled in the trial. The primary endpoint of the phase Ib portion was safety and tolerability, and that of the phase II was CR rate. In the phase II section, 80 patients with R/R DLBCL deemed transplant-ineligible or with relapsed post APSCT were randomized in a 1:1 ratio to receive either the triplet P-BR or BR alone, every 21 days, for a total of six cycles. Polatuzumab vedotin was initially given intravenously at a dose of $2.4 \mathrm{mg} / \mathrm{kg}$, subsequently reduced to $1.8 \mathrm{mg} / \mathrm{kg}$ because of safety concerns mostly related to peripheral neuropathy, administered on day 2 of the first cycle, and on the first day of the remaining cycles. Rituximab was given at $375 \mathrm{mg} / \mathrm{m}^{2}$ on day 1 of each cycle and bendamustine at $90 \mathrm{mg} / \mathrm{m}^{2} \mathrm{IV}$ on days 1 and 2 of each cycle. The randomized patients had a median age of 67 years (range, 33-86) for the P-BR cohort and 71 years (range, 30-84) for the BR arm. Enrolled patients had failed a median of two prior therapies, with the majority being refractory to the latest therapy (75\% in P-BR and $85 \%$ in BR) including APSCT (25\% in P-BR and $15 \%$ in BR).

In the most recently published analysis of the randomized data, the triplet combination ( $n=40$ patients) was more favorable than $\mathrm{BR}(n=40$ patients) with regards to $\mathrm{CR}$ rate $(40 \%$ versus $18 \%$; $p=0.026)$ and OR (45\% versus $18 \%$; $p$-value not reported) at treatment completion and a median follow-up of 22 months.
The polatuzumab-containing arm also resulted in numerically longer DoR than the comparator regimen (13 versus 8 months), mostly among patients who achieved CR or PR. Notably, seven patients treated with $\mathrm{P}-\mathrm{BR}$ still record ongoing responses beyond 20 months, including one patient bridged to allogeneic stem cell transplantation (ASCT). Although not powered to detect a survival difference, the triplet regimen was also associated with a longer median PFS (7.6 versus 2.0 months; $p<0.0001)$ as well as OS (12.4 versus 4.7 months; $p=0.0023$ ) compared to $\mathrm{BR}$, irrespective of age ( $<65$ versus $\geq 65$ years), prior therapy, the COO or MYC/BCL2 double-expressor status.

Based on this analysis, polatuzumab vedotin is currently approved for the use in patients with R/R DLBCL who have received at least two prior therapies, including APSCT, or those who are transplant ineligible.

\subsection{Ongoing Trials}

Several ongoing trials are currently evaluating the safety and efficacy of polatuzumab vedotin in combination with chemotherapy or MoAbs, as frontline treatment or in the R/R setting (Table 1).

One important study is the POLARIX phase III, randomized, double-blind trial comparing the efficacy and safety of standard $\mathrm{R}-\mathrm{CHOP}$ to polatuzumab vedotin $(1.8 \mathrm{mg} / \mathrm{kg})$ plus $\mathrm{R}-\mathrm{CHP}$ for the frontline treatment of DLBCL [28]. Patients received six or eight 21-day cycles of treatment and available data of 66 patients at a median study duration of 21.5 months suggest that $95 \%$ of responses were still ongoing at 12 months. Published results also indicate that the 12- and 24-month PFS rates were 91\% and $83 \%$, while the 12 -month OS rate was $94 \%$. On pre-specified analyses, PFS and responses did not correlate with CD79b expression or with the DLBCL COO, as well as with MYC/BCL2 double-expression status (for PFS). Exploratory analyses also showed that the number of planned treatment cycles, the type of immunotherapy agent, or the International Prognostic Index score did not affect PFS [29].

\subsection{Adverse Events: Management and Practice Pearls}

Studies involving polatuzumab vedotin displayed an acceptable safety profile. When used as monotherapy, treatment-emergent AEs (TEAEs) were generally of grade 1-2 [23]. However, those who received polatuzumab vedotin at $2.4 \mathrm{mg} / \mathrm{kg}$ had more grade $3 / 4$ TEAEs including neutropenia (40\%; leading to delay in treatment), anemia (11\%) and peripheral sensory neuropathy (9\%), the latter leading to discontinuation in $24 \%$ of patients. Serious TEAEs included diarrhea and lung infection. In the setting of combinatorial strategies, AEs were similar, whether polatuzumab vedotin-immunotherapy was used with or without concomitant chemotherapy. For instance, in the GO29365 study, AEs of all grades occurred most frequently ( $>20 \%$ incidence) with P-BR triplet therapy at $\geq 5 \%$ greater incidence than with $\mathrm{BR}$, including neutropenia (49\% versus $44 \%$ ), thrombocytopenia (49\% versus $33 \%$ ), anemia ( $47 \%$ versus $28 \%$ ), peripheral neuropathy ( $40 \%$ versus $8 \%$ ) which resolved in the majority of patients, diarrhea ( $38 \%$ versus $28 \%$ ), and pneumonia (22\% versus $15 \%$ ), while $7 \%$ died from toxicities within 90 days of receiving their last treatment. 
Table 1 Ongoing trials involving polatuzumab vedotin-piiq in DLBCL

\begin{tabular}{|c|c|c|c|c|}
\hline $\operatorname{Drug}(s)$ & $\begin{array}{l}\text { Clinical } \\
\text { phase }\end{array}$ & Indications/endpoints & Status & Identifiers \\
\hline Polatuzumab vedotin + DA-EPCH-R & I & $\begin{array}{l}\text { Previously untreated aggressive large B-cell } \\
\text { lymphoma; safety and toxicity }\end{array}$ & Not yet recruiting & NCT04231877 \\
\hline Polatuzumab vedotin + R-CHP & III & $\begin{array}{l}\text { Previously untreated diffuse large B-cell } \\
\text { lymphoma; PFS }\end{array}$ & Recruiting & NCT03274492 \\
\hline Polatuzumab vedotin + Mosunetuzumab & $\mathrm{Ib} / \mathrm{II}$ & R/R FL or DLBCL; Safety and efficacy DLT MTD & Recruiting & NCT03671018 \\
\hline Polatuzumab vedotin $+\mathrm{BR}$ & III & $\begin{array}{l}\text { Transplant ineligible R/R DLBCL; Complete } \\
\text { remission rate }\end{array}$ & Not yet recruiting & NCT04236141 \\
\hline Polatuzumab vedotin with R-GemOx & III & R/R DLBCL; Safety and OS & Recruiting & NCT04182204 \\
\hline $\begin{array}{l}\text { Polatuzumab vedotin + Venetoclax with } \\
\text { Rituximab or Obinituzumab }\end{array}$ & $\mathrm{Ib} / \mathrm{II}$ & $\begin{array}{l}\mathrm{R} / \mathrm{R} \text { FL or R/R DLBCL; Complete remission } \\
\text { rate and safety }\end{array}$ & Active, not recruiting & NCT02611323 \\
\hline $\begin{array}{l}\text { Polatuzumab vedotin + Lenalidomide with } \\
\text { Rituximab or Obinituzumab }\end{array}$ & $\mathrm{Ib} / \mathrm{II}$ & $\begin{array}{l}\mathrm{R} / \mathrm{R} \text { FL or R/R DLBCL; Complete remission } \\
\text { rate and safety }\end{array}$ & Active, not recruiting & NCT02600897 \\
\hline Polatuzumab vedotin + Glofitamab & $\mathrm{Ib}$ & R/R non-Hodgkin B-cell lymphoma; DLTs & Recruiting & NCT03533283 \\
\hline Polatuzumab vedotin + R mini-CHP & III & Previously untreated DLBCL; PFS & Not yet recruiting & NCT04332822 \\
\hline $\begin{array}{l}\text { Polatuzumab vedotin + Bendamustine with } \\
\text { Rituximab or Obinituxumab }\end{array}$ & $\mathrm{Ib} / \mathrm{II}$ & $\begin{array}{l}\text { R/R FL or DLBCL; safety and tolerability, } \\
\text { CR rate }\end{array}$ & Active, not recruiting & NCT02257567 \\
\hline Polatizumab vedotin $+\mathrm{CHP}$ and Mosunetuzumab & $\mathrm{Ib} / \mathrm{II}$ & $\begin{array}{l}\mathrm{R} / \mathrm{R} \text { non-Hodgkin B-cell lymphoma; Safety } \\
\text { and complete response rate }\end{array}$ & Recruiting & NCT03677141 \\
\hline
\end{tabular}

BR, bendamustine rituximab; DA-EPCH-R, dose-adjusted-etoposide, prednisone, vincristine (Oncovin), cyclophosphamide, doxorubicin-rituximab; DLBCL, diffuse large B-cell lymphoma; DLT, dose-limiting toxicities; FL, follicular lymphoma; MTD, maximum tolerated dose; OS, overall survival; PFS, progression free survival; R-CHP, rituximabcyclophosphamide, doxorubicin, prednisone; R-GemOx, rituximab, gemcitabine, oxaliplatin.

Importantly, the rate of febrile neutropenia was similar in the two cohorts (10\% P-BR, 13\% BR).

The peripheral neuropathy seen with polatuzumab-based therapy is commonly dose- and duration-dependent, as seen with other ADC-containing MMAE. These DLBCL patients have generally received prior platinum and/or vinca alkaloids therapy, and those who present with grade $\geq 2$ neuropathy were therefore not eligible to receive polatuzumab. Nevertheless, for patients who develop polatuzumab-induced grade $\geq 2$ neuropathy at the dose of $1.8 \mathrm{mg} / \mathrm{kg}$, it is recommended to hold therapy until resolution to grade $\leq 1$, and then resume polatuzumab with the upcoming cycles at a dose of $1.4 \mathrm{mg} / \mathrm{kg}$. Treatment discontinuation is advised if neuropathy is of grade 4 , if it does not resolve to grade $\leq 1$ by 2 weeks of holding polatuzumab or if it recurs to grade $\geq 2$ despite dose reduction.

Premedication with an antihistamine and antipyretics is advised with P-BR and prophylaxis for Pneumocystis jirovecii and herpesviruses is also recommended. The first dose of polatuzumab vedotin is usually administered over $90 \mathrm{~min}$, while subsequent doses could be given over $30 \mathrm{~min}$ if tolerated. Despite premedications, infusion reactions still occur in $7 \%$ of the P-BR cohort, mostly of low-grade.

It is important to note that polatuzumab vedotin is potentially immunogenic. In the phase Ib/II study GO29365, eight of 134 (6\%) recipients across all polatuzumab-containing arms recorded anti-polatuzumab vedotin antibodies, and a total of 14 of 536 (2.6\%) evaluable recipients across all clinical trials. However, the relatively small percentage of these auto-antibodies preclude further conclusions on their potential impact on the safety and efficacy of polatuzumab-based regimens.

\subsection{Real-World Data}

Data from real-life experience are of great need to confirm the favorable outcomes reported in clinical trials. In this regard, very recent data by Dimou et al. [30] on $61 \mathrm{R} / \mathrm{R}$ DLBCL patients who received polatuzumab with BR showed median PFS, OS and DoR of $4,8.5$, and 8.5 months, respectively. The ORR was $43 \%$, including $25 \%$ CR and $18 \%$ PR. Notably, one patient was able to proceed with CAR T-cell therapy and two were bridged to ASCT. With the aforementioned findings and a rate of $55 \%$ of patients experiencing a grade $\geq 3 \mathrm{AE}$, this report confirms the combination of $\mathrm{P}-\mathrm{BR}$ as a viable and promising option for R/R DLBCL through a real-world experience.

\subsection{Perspective}

Several trials have strongly placed polatuzumab vedotin in the treatment armamentarium of DLBCL. More specifically, the aforementioned results of trial have established the triplet P-BR as a novel and effective treatment strategy for heavily pretreated and transplantation-ineligible DLBCL patients. Nevertheless, GO29365 did not include patients with transformed FL or double- and triplehit lymphoma, therefore questioning the efficacy of polatuzumab in this specific population. It is, however, noteworthy to mention that GO29365 is a small trial underpowered to confirm any survival benefit with the triplet and that $\mathrm{BR}$, the comparator arm, has marginal clinical activity. POLARGO (NCT04182204) is a crucial ongoing phase III trial randomizing DLBCL patients to rituximab, gemcitabine, and oxaliplatin alone or in combination with polatuzumab vedotin (Table 1), whereby the comparator arm is considered as more effective than BR; this study should clarify the role of polatuzumab vedotin in APSCT-eligible patients. Furthermore, as only $25 \%$ of patients in the P-BR cohort of the GO29365 trial had received prior APSCT and none had prior CAR-T, the actual role of the combination in this subgroup remains undefined. Finally, one must consider the potential negative impact on stem cell mobilization and CAR-T preparation of a prolonged therapy with bendamustine in heavily pretreated patients, thereby questioning the role of $\mathrm{P}-\mathrm{BR}$ as bridging therapy in this situation. These concerns 
might not remain legit if bendamustine is used following apheresis for cell collection, especially in the case of CAR-T cell therapy. In parallel, bendamustine could possess deep lymphodepleting effects that might be beneficial for CAR-T cell expansion.

Given the molecular and biological significance of CD79b in DLBCL, the suboptimal outcomes in patients with high-risk disease, as well as the lack of any role for maintenance therapy in LBCL, it could be potentially beneficial to evaluate this ADC as maintenance therapy following first CR or APSCT for R/R patients with aggressive disease. The results of ongoing clinical trials are eagerly awaited and will help confirm the efficacy of polatuzumab vedotin at various stages of the disease and with different combinations.

\section{CONFLICTS OF INTEREST}

The authors declare they have no conflicts of interest.

\section{AUTHORS' CONTRIBUTION}

$\mathrm{RA}$ and $\mathrm{AB}$ created the concept of this article. RA wrote the article. All authors reviewed and edited the manuscript.

\section{FUNDING}

No financial support was provided.

\section{REFERENCES}

[1] Teras LR, DeSantis CE, Cerhan JR, Morton LM, Jemal A, Flowers CR. 2016 US lymphoid malignancy statistics by World Health Organization subtypes. CA Cancer J Clin 2016;66;443-59.

[2] Coiffier B. State-of-the-art therapeutics: diffuse large B-cell lymphoma. J Clin Oncol 2005;23;6387-93.

[3] Habermann TM, Weller EA, Morrison VA, Gascoyne RD, Cassileth PA, Cohn JB, et al. Rituximab-CHOP versus CHOP alone or with maintenance rituximab in older patients with diffuse large B-cell lymphoma. J Clin Oncol 2006;24;3121-7.

[4] Liu Y, Barta SK. Diffuse large B-cell lymphoma: 2019 update on diagnosis, risk stratification, and treatment. Am J Hematol 2019;94;604-16.

[5] Karlin L, Coiffier B. Improving survival and preventing recurrence of diffuse large B-cell lymphoma in younger patients: current strategies and future directions. Onco Targets Ther 2013;6;289-96.

[6] Crump M, Neelapu SS, Farooq U, Van Den Neste E, Kuruvilla J, Westin J, et al. Outcomes in refractory diffuse large B-cell lymphoma: results from the international SCHOLAR-1 study. Blood 2017;130;1800-8.

[7] Gisselbrecht C, Van Den Neste E. How I manage patients with relapsed/refractory diffuse large B cell lymphoma. Br J Haematol 2018;182;633-43.

[8] Schuster SJ, Bishop MR, Tam CS, Waller EK, Borchmann P, McGuirk JP, et al. Tisagenlecleucel in adult relapsed or refractory diffuse large B-cell lymphoma. N Engl J Med 2019;380;45-56.

[9] Chavez JC, Bachmeier C, Kharfan-Dabaja MA. CAR T-cell therapy for B-cell lymphomas: clinical trial results of available products. Ther Adv Hematol 2019;10;2040620719841581.
[10] Hedrich WD, Fandy TE, Ashour HM, Wang H, Hassan HE. Antibody-drug conjugates: pharmacokinetic/pharmacodynamic modeling, preclinical characterization, clinical studies, and lessons learned. Clin Pharmacokinet 2018;57;687-703.

[11] Polson AG, Yu SF, Elkins K, Zheng B, Clark S, Ingle GS, et al. Antibody-drug conjugates targeted to CD79 for the treatment of non-Hodgkin lymphoma. Blood 2007;110;616-23.

[12] Li C, Zhang C, Li Z, Samineni D, Lu D, Wang B, et al. Clinical pharmacology of vc-MMAE antibody-drug conjugates in cancer patients: learning from eight first-in-human Phase 1 studies. mAbs 2020;12;1699768.

[13] Dal Porto JM, Gauld SB, Merrell KT, Mills D, Pugh-Bernard AE, Cambier J. B cell antigen receptor signaling 101. Mol Immunol 2004;41;599-613.

[14] Martin AW. Immunohistology of non-Hodgkin lymphoma. In: Dabbs DJ, editor. Diagnostic immunohistochemistry: theranostic and genomic applications. 3rd ed. Philadelphia: Saunders Elsevier; 2010, pp. 156-88.

[15] Chu PG, Arber DA. CD79: a review. Appl Immunohistochem Mol Morphol 2001;9;97-106.

[16] Winkler TH, Mårtensson IL. The role of the pre-B cell receptor in B cell development, repertoire selection, and tolerance. Front Immunol 2018;9;2423.

[17] Dornan D, Bennett F, Chen Y, Dennis M, Eaton D, Elkins K, et al. Therapeutic potential of an anti-CD79b antibody-drug conjugate, anti-CD79b-vc-MMAE, for the treatment of non-Hodgkin lymphoma. Blood 2009;114;2721-9.

[18] Pfeifer M, Zheng B, Erdmann T, Koeppen H, McCord R, Grau M, et al. Anti-CD22 and anti-CD79B antibody drug conjugates are active in different molecular diffuse large B-cell lymphoma subtypes. Leukemia 2015;29;1578-86.

[19] Tiwari AA, Ayello J, van de Ven C, Kurien L, Barth MJ, Cairo MS. Antibody drug conjugates (anti-CD79b-vc-MMAE, Polatuzumab Vedotin) exhibit enhanced cell death targeted to CD79b+ Burkitt lymphoma (BL) and primary mediastinal large B-cell lymphoma (PMBL). Cancer Res 2016;76;579.

[20] Tiwari AA, Edani D, Azmy C, Ayello J, Klein C, Cairo MS. Enhanced in vitro/in vivo cytotoxicity against Burkitt lymphoma/ primary mediastinal large B cell lymphoma by polatuzumab vedotin (hu-anti-CD79b-vc-MMAE, PV) alone or in combination with obinutuzumab [abstract no. 1788]. Cancer Res 2018;78.

[21] Amin DN, Oeh J, Zindal A, Musick L, Hirata J, Mobasher M, et al. Anti-tubulin antibody drug conjugates potentiate venetoclax activity in non-Hodgkin lymphoma by targeting MCL-1 [abstract no. 880]. Cancer Res 2018;78.

[22] Tiwari AA, Edani D, Ayello J, Klein C, Lee D, Cairo M. Polatuzumab vedotin alone or in combination with obinutuzumab synergistically enhances in vitro cytotoxicity and cytokine release against CD20+/CD79b + burkitt lymphoma (BL)/primary mediastinal large B cell lymphoma (PMBL) [abstract]. Blood $2017 ; 130 ; 1540$.

[23] Palanca-Wessels MC, Czuczman M, Salles G, Assouline S, Sehn LH, Flinn I, et al. Safety and activity of the anti-CD79B antibodydrug conjugate polatuzumab vedotin in relapsed or refractory B-cell non-Hodgkin lymphoma and chronic lymphocytic leukaemia: a phase 1 study. Lancet Oncol 2015;16;704-15.

[24] Hatake K, Kinoshita T, Terui Y, Yokoyama M, Maruyama D, Makita S, et al. A phase I pharmacokinetic and safety study of polatuzumab vedotin in Japanese patients with relapsed/refractory b-cell non-Hodgkin lymphoma: a comparison with nonJapanese DCS4968g study. J Clin Oncol 2016;34;e19070. 
[25] Morschhauser F, Flinn IW, Advani R, Sehn LH, Diefenbach C, Kolibaba K, et al. Polatuzumab vedotin or pinatuzumab vedotin plus rituximab in patients with relapsed or refractory non-Hodgkin lymphoma: final results from a phase 2 randomised study (ROMULUS). Lancet Haematol 2019;6;e254-e65.

[26] Phillips T, Brunvand M, Chen A, Press O, Essell J, Chiappella A, et al. Polatuzumab vedotin combined with obinutuzumab for patients with relapsed or refractory non-Hodgkin lymphoma: preliminary safety and clinical activity of a phase Ib/II study. Blood 2016;128;622.

[27] Sehn LH, Herrera AF, Flowers CR, Kamdar MK, McMillan A, Hertzberg M, et al. Randomized phase 2 trial of polatuzumab vedotin (pola) with bendamustine and rituximab (BR) in relapsed/ refractory (r/r) FL and DLBCL. J Clin Oncol 2018;36;7507.
[28] Tilly H, Flowers C, Friedberg JW, Herbaux C, Morschhauser F, Sehn LH, et al. POLARIX: a phase 3 study of polatuzumab vedotin (pola) plus R-CHP versus R-CHOP in patients (pts) with untreated DLBCL. Hematol Oncol 2019;37;68-70.

[29] Tilly H, Morschhauser F, Bartlett NL, Mehta A, Salles G, Haioun C, et al. Polatuzumab vedotin in combination with immunochemotherapy in patients with previously untreated diffuse large B-cell lymphoma: an open-label, non-randomised, phase 1b-2 study. Lancet Oncol 2019;20;998-1010.

[30] Dimou M, Papageorgiou SG, Stavroyianni N, Katodritou E, Tsirogianni M, Kalpadakis C, et al. Real-life experience with the combination of polatuzumab vedotin, rituximab and bendamustine in aggressive B-cell lymphomas. Hematol Oncol 2021 [Epub ahead of print]. 